\title{
CONFORMAL INVARIANTS OF MINKOWSKI SPACE
}

\author{
JACK MORAVA
}

\begin{abstract}
The conformal invariant defined for compact Riemannian manifolds by Yamabe is generalized to pseudo-Riemannian manifolds and is shown to be nontrivial for Minkowski space. We also make some elementary remarks about generalizations of Yamabe's equation to sections of vector bundles, as have been studied by physicists concerned with Goldstone bosons and the Higgs mechanism.
\end{abstract}

... But I will try to show you by means of geometrical proofs ... that, of the numbers named by me and given in the work which I sent to [my funding agency], some exceed not only the number of the mass of sand equal in magnitude to the earth filled up in the way described, but also that of a mass equal in magnitude to the universe...

Archimedes, the Sandreckoner

If $(X, g)$ is a compact Riemannian manifold of dimension at least three, then the infimum of the set

$$
\left\{\left(\left(-\Delta_{g}+\frac{1}{4} \frac{n-2}{n-1} R(g)\right) b, b\right) \mid b \in L_{1}^{2}\left(X, \operatorname{vol}_{g}\right),\|b\|=1\right\}
$$

of real numbers, $\|\cdot\|$ denoting the Lebesgue $2 n /(n-2)$-norm, is a remarkable invariant of the conformal diffeomorphism class of $(X, g)$, cf. [20]. As an example, we cite

Proposition 1. When $(X, g)$ is the usual $n$-sphere, the infimum defined above has the value

$$
\frac{1}{4} n(n-2) \omega_{n}^{2 / n}
$$

(e.g. $\frac{4}{3} \pi \cdot 6^{1 / 2}$ when $n=4$ ) where $\omega_{n}$ is the area of the unit sphere in $\mathbf{R}^{n+1}$.

See [2] for the proof.

Note that there is a natural embedding

$$
L_{r}^{p}\left(X, \operatorname{vol}_{g}\right) \rightarrow L_{s}^{q}\left(X, \operatorname{vol}_{g}\right)
$$

of Sobolev spaces if $r-n p^{-1} \geqslant s-n q^{-1}$ [7], e.g. if $p=2, r=1, s=0$, and $q \leqslant 2 n /(n-2)$; so the definition above makes sense.

More generally, any critical value of the quadratic form

$$
b \mapsto\left(Y_{g} b, b\right), \quad Y_{g}=-\Delta_{g}+\frac{1}{4} \frac{n-2}{n-1} R(g)
$$

on the unit sphere in the Lebesgue space $L^{2 n /(n-2)}\left(X, \mathrm{vol}_{g}\right)$ is a conformal invariant, provided that $b$ is understood as a section of the line bundle of densities of conformal weight $\frac{1}{2} n-1$. (We use the normalization convention of physics, in which

Received by the editors August 14, 1984 and, in revised form, October 16, 1984.

1980 Mathematics Subject Classification. Primary 35P30, 53A50, 53C50, 53C80, 58C40. 
densities $\phi$ of weight $w$ have norm

$$
\|\phi\|=\left[\int_{X}|\phi|^{n / w}\right]^{w / n}
$$

defined without reference to any Riemannian metric.)

The purpose of this note is to draw attention to this conformal spectrum of critical values of $Y_{g}$ on the unit sphere in the space of $\left(\frac{1}{2} n-1\right)$-densities, as a substitute for the lack of any conformally natural Hilbert space representation of $Y_{g}$, and to show by calculation that such invariants can be defined more generally, e.g. for manifolds noncompact or pseudo-Riemannian or both. Note that a real critical point ${ }^{1}$ of the Yamabe functional can be interpreted to be a conformal deformation of $g$ to $\bar{g}=|b|^{4 /(n-2)} g$ such that $\operatorname{vol}_{\bar{g}}$ is a probability measure and $R(\bar{g})$ is almost certainly constant.

I am indebted to Tom Branson for drawing my attention to classical Euclidean space:

Proposition 2. The conformal spectrum of $\mathbf{R}^{n}$ is nonempty and, in particular, contains $\frac{1}{4} n(n-2) \omega_{n}^{2 / n}$.

Proof. The problem of extremizing the Dirichlet form on the unit sphere in $L^{2 n /(n-2)}\left(\mathbf{R}^{n}\right)$ leads to the Lagrange multiplier functional

$$
t, b \mapsto(-\Delta b, b)+t\left(\|b\|^{2 n /(n-2)}-1\right),
$$

with critical points verifying

$$
-\Delta b+(t n /(n-2))|b|^{4 /(n-2)} b=0, \quad\|b\|=1 ;
$$

but it is well known that $b_{0}(x)=\left(1+\|x\|^{2}\right)^{-\frac{1}{2}(n-2)}$ satisfies

$$
-\Delta b_{0}+n(n-2) b_{0}^{(n+2) /(n-2)}=0
$$

(cf. [15] for some related examples), and it is easy to calculate that

$$
\left\|b_{0}\right\|^{2 n /(n-2)}=\frac{1}{2} B\left(\frac{1}{2} n, \frac{1}{2} n\right) \omega_{n-1}, \quad \text { with } \omega_{n-1}=2 \pi^{\frac{1}{2} n} / \Gamma\left(\frac{1}{2} n\right),
$$

where $B$ is Euler's beta-function. The assertion then follows from the duplication formula for the gamma function, and rescaling.

For pseudo-Riemannian manifolds of hyperbolic signature we write $\square_{g}$ to denote the Laplace-Beltrami operator; in this notation our main result is then

PROPOSITION 3. The set of critical values of an appropriate closure of the densely defined quadratic form $b \mapsto(\square b, b)$ restricted to the unit sphere in the Lebesgue space $L^{2 n /(n-2)}$ of Minkowski space is nonempty, and in particular contains $\left(\frac{1}{2} n-1\right)^{2}\left(\pi \omega_{n-1}\right)^{\frac{1}{2} n}$ e.g. $\left(2 \pi^{3}\right)^{\frac{1}{2}}$ when $n=4$.

Proof. As in the preceding proposition it will be enough to exhibit a critical point associated to the above critical value; it will turn out that this kind of critical point is well known in other contexts, as we will see below. Taking the domain of the form to

\footnotetext{
${ }^{1}$ Critical points correspond to conformal "vacuum states", of N. D. Birrell and P. C. W. Davies, Quantum fields in curved space-time, Cambridge University Press, 1982.
} 
consist of those elements of $L^{2 n \lambda n-2)}\left(\mathbf{R}^{n}\right)$ for which the Dirichlet integral $\int|d b|^{2}$ vol is finite (i.e. calculated with respect to the usual Euclidean metric, cf. the homogeneous Sobolev theorem of [14]), an elementary computation completes the argument:

LEMMA. There is a smooth, nowhere-vanishing real-valued solution $u$ to the nonlinear Klein-Gordon equation

$$
\square u-(n-2)^{2} u^{(n+2) /(n-2)}=0
$$

on Minkowski space, with $u$ of Lebesgue class $L^{2 n /(n-2)}$.

Proof. Let $v=t+r, w=t-r$, with $r^{2}=x^{2}+y^{2}+z^{2}$, be advanced and retarded coordinates; then $u=\left[\left(1+v^{2}\right)\left(1+w^{2}\right)\right]^{-\frac{1}{4}(n-2)}$ is such a solution, with

$$
\|u\|^{2 n /(n-2)}=2^{-n} \pi \omega_{n-1}
$$

the proposition above follows immediately by rescaling. Indeed, in advanced and retarded coordinates we can write the wave operator as

$$
\square u=4 \frac{\partial^{2} u}{\partial v \partial w}-\frac{2(n-2)}{v-w}\left(\frac{\partial u}{\partial v}-\frac{\partial u}{\partial w}\right),
$$

and the assertion that $u$ is a solution follows immediately. Now

$$
\|u\|^{2 n /(n-2)}=2^{-n} \omega_{n-2} \int_{\mathbf{R}^{2}} \frac{|v-w|^{n-2}}{\left[\left(1+v^{2}\right)\left(1+w^{2}\right)\right]^{\frac{1}{2} n}} d v d w
$$

can be evaluated as

$$
\frac{2^{-1-2 k} \pi^{k+\frac{1}{2}}}{\Gamma\left(k+\frac{1}{2}\right)} \sum_{i=0}^{i=k}\left(\begin{array}{c}
2 k \\
2 i
\end{array}\right) B(i+1 / 2, k-i+1 / 2)^{2},
$$

where $n=2 k+2$. Using the duplication formula, this sum becomes

$$
2^{-n} \omega_{n-1} \sum_{i=0}^{i=k} \frac{\Gamma(i+1 / 2) \Gamma(k-i+1 / 2)}{\Gamma(i+1) \Gamma(k-i+1)}
$$

the assertion that the sum over gamma-functions equals $\pi$ is equivalent to the identity

$$
2^{2 k-2}=\left(\begin{array}{c}
2 k-1 \\
k
\end{array}\right)+\sum_{i=1}^{i=k-1}\left(\begin{array}{c}
2 i-1 \\
i
\end{array}\right)\left(\begin{array}{c}
2 k-2 i-1 \\
k-i
\end{array}\right),
$$

which can be verified by noting that the function

$$
g(x)=\sum_{k=1}^{\infty}\left(\begin{array}{c}
2 k-1 \\
k
\end{array}\right) x^{k}=1 / 2\left((1-4 x)^{-\frac{1}{2}}-1\right)
$$

satisfies the equation

$$
g(x)^{2}+g(x)=x(1-4 x)^{-1}=\sum_{k=1}^{\infty} 2^{2 k-2} x^{k} .
$$

These functions become familiar in more traditional coordinates; following Penrose [18] via Hawking and Ellis, we can write

$$
\tau=\operatorname{Arctan} v+\operatorname{Arctan} w, \quad \rho=\operatorname{Arctan} v-\operatorname{Arctan} w,
$$


so that the quadratic differential

$$
d s_{E}^{2}(\tau, \rho, \theta, \phi)=d \tau^{2}-d \rho^{2}-\sin ^{2} \rho\left(d \theta^{2}+\sin ^{2} \theta d \phi^{2}\right)
$$

of Einstein's static universe (as parametrized in $[12, \S 5.1]$ ) takes the form

$$
u(r, t)^{2}\left[d t^{2}-d r^{2}-r^{2}\left(d \phi^{2}+\sin ^{2} \theta d \phi^{2}\right)\right]=u^{2}(r, t) d s_{\text {Minkowski }}^{2}(t, r, \theta, \phi)
$$

in Minkowski space. Similarly, in the anti-de Sitter universe [12, §5.2] the quadratic differential is

$$
\begin{aligned}
d s_{-}^{2}(\tau, \chi, \theta, \phi) & =\cosh ^{2} \chi d \tau^{2}-d \chi^{2}-\sinh ^{2} \chi\left(d \theta^{2}+\sin ^{2} \theta d \phi^{2}\right) \\
& =\cosh ^{2} \chi d s_{E}^{2}(\tau, \rho, \theta, \phi),
\end{aligned}
$$

with $\chi=2 \operatorname{Arctan} \exp \rho-\frac{1}{2} \pi$; hence $d s_{-}^{2}(\tau, \chi, \theta, \phi)=\Phi_{-}^{2} d s_{\text {Minkowsi }}^{2}(t, r, \theta, \phi)$ with

$$
\Phi_{-}=2 \cosh \chi \cdot \cos \left(\frac{1}{2}(\tau+\rho)\right) \cos \left(\frac{1}{2}(\tau-\rho)\right)=2\left(1-\langle x\rangle^{2}\right)^{-1},
$$

while in the de Sitter universe we have

$$
\begin{aligned}
d s_{+}^{2}(\chi, \rho, \theta, \phi) & =d \chi^{2}-\cosh ^{2} \chi\left[d \rho^{2}+\sin ^{2} \rho\left(d \theta^{2}+\sin ^{2} \theta d \phi^{2}\right)\right] \\
& =\Phi_{+}^{2} d s_{\text {Minkowski }}^{2}(t, r, \theta, \phi),
\end{aligned}
$$

with

$$
\Phi_{+}=2\left(1+\langle x\rangle^{2}\right)^{-1}, \quad t=2 \operatorname{Arctan} \exp \chi-\frac{1}{2} \pi
$$

denoting by $\langle x\rangle^{2}=t^{2}-r^{2}$ the Minkowski distance function. It is easy to check that $\Phi_{ \pm}$are solutions to Yamabe's equation, in appropriate domains; but $\left\|\Phi_{ \pm}\right\|=\infty$. Similarly, the Robertson-Walker, or "Friedmon", metrics [11] $d s^{2}=d t^{2}-S(t)^{2} d \sigma^{2}$, in which $\dot{S}^{2}=\bar{S}^{1} \cdot\left(S_{0}-S\right)$, are conformally diffeomorphic, via the map

$$
\left(t, x_{1}, x_{2}, x_{3}\right) \mapsto\left(\mathbf{t}(t), x_{1}, x_{2}, x_{3}\right), \quad \mathbf{i}=\bar{S}^{1},
$$

to suitable subuniverses of the static universe, which thus plays a role analogous to the upper half-plane, cf. [12, §5.3].

A very clear account of the solution to Yamabe's problem used in Proposition 3 can be found in $\S 3.7$ of B. Crsted's paper [17]; see also [19]. It may also be worth remarking, because of the problem of infrared divergences, that the function studied in Proposition 2 is not in $L^{2}$ if $n$ is less than five; nor is the function $u$ of the lemma, cf. [8].

Now equations much like Yamabe's but complexified seem to be of great interest to physicists interested in symmetry-breaking, cf. in particular [10], or more generally $[1,5,6,9,16,21, \ldots]$; though it is often difficult to tell what is true in dimension four and what is true in all dimensions. As a concluding unscientific postscript, note that if $\phi=b e^{i \theta}$ with $b$ in $L_{1}^{2}\left(X, \operatorname{vol}_{g} ; \mathbf{R}\right)$ and $\theta$ in $L_{1}^{n / 4}\left(X, \operatorname{vol}_{g} ; \mathbf{R}\right)$, then the expression

$$
\int\left[|d b|_{g}^{2}+b^{2}|d \theta|_{g}^{2}\right] d \text { vol }
$$

(which is just the Dirichlet form of $\phi$ if both $b$ and $\theta$ are smooth) is at least finite, which suggests that critical points of the Yamabe functional be sought among 
solutions of the Euler equations

(i) $|\operatorname{det} g|^{-1 / 2}\left(|\operatorname{det} g|^{1 / 2} g^{i k} b^{2} \theta_{, k}\right)_{, i}=0$,

(ii) $\left(\square_{g}+\frac{1}{4}((n-2) /(n-1)) R(g)+|d \theta|_{g}^{2}+\right.$ constant $\left.|b|^{4 /(n-2)}\right) b=0$.

With $\bar{g}=|b|^{4 /(n-2)} g$ equation (ii) can be rewritten

(ii*) $R(\bar{g})=$ constant $-4((n-1) /(n-2))|d \theta|_{\bar{g}}^{2}$ almost everywhere w.r.t. $\bar{g}$, and equation (i) becomes

(i*) $\square_{\bar{g}} \theta=0$ a.e.

A good example is

$$
\phi\left(x_{0}, \ldots, x_{n-1}\right)=e^{i k x_{0}} b\left(x_{1}, \ldots, x_{n-1}\right)
$$

the Sobolev theorems then lead to strong existence for the Helmholtz equation

$$
\left[-\Delta_{n-1}+k^{2}+\text { constant } \cdot|b|^{4 /(n-2)}\right] b=0,
$$

cf. [3, 13]. But although this solution has finite energy density, it is not in $L^{2 n /(n-2)}\left(\mathbf{R}^{n}\right)$. For similar reasons, zero cannot be in the conformal spectrum of Minkowski space.

AcKnowledgment. Conversation and correspondence with Jacob Sturm, Herb Bernstein, Gabor Domokos, Evans Harrell, Ed Nelson, Louis Nirenberg, and the editors have been of invaluable help in this work, and I want to acknowledge this with thanks.

\section{REFERENCES}

1. M. Aizenmann, Proof of the triviality of $\phi_{d}^{4}$ theory and some mean-field features of Ising models for $d>4$, Phys. Rev. Lett. 47 (1981), 1-4.

2. T. Aubin, Equations differentielles non lineaires et probleme de Yamabe, J. Math. Pures Appl. 55 (1976), 269-296.

3. H. Berestycki and P. L. Lions, Existence d'ondes solitaires dans des problèmes nonlinéaires du type Klein-Gordon, C. R. Acad. Sci. Paris Sér. A-B 288 (1979), 395-398.

4. T. Branson, Differential operators canonically associated to a conformal structure, preprint, Purdue University, 1984.

5. L. Brown and J. Collins, Dimensional renormalization of scalar field theory in curved space-time, Ann. Phys. 130 (1980), 215-248.

6. D. Brydges, What is quantum field theory?, Bull. Amer. Math. Soc. (N.S.) 8 (1983), 31-41.

7. A. P. Calderón, Lebesgue spaces of differentiable functions and distributions, Partial Differential Equations (C. B. Morrey, ed.), Proc. Sympos. Pure Math., vol. 4, Amer. Math. Soc., Providence, R.I., 1961, pp. 33-49.

8. L. Castell, Exact solutions of the $\lambda \phi^{4}$ theory, Phys. Rev. D 6 (1972), 536-538.

9. F. Cooper and G. Venturi, Cosmology and broken scale invariance, Phys. Rev. D 24 (1981), $3338-3340$.

10. G. Domokos, M. M. Janson, S. Kovesi-Domokos, Possible cosmological origin of spontaneous symmetry-breaking, Nature 257 (1975), 203-204.

11. A. Einstein, The meaning of relativity, Princeton Univ. Press, Princeton, N.J., 1945.

12. G. Ellis and S. Hawking, The large-scale structure of space-time, Cambridge Univ. Press, 1973.

13. J. Goldstone, Field theories with superconductor solutions, Nuovo Cimento 19 (1961), 154-164.

14. V. F. Kovalenko, M. A. Perelmuter and Y. Semenov, Schrodinger operators with $L_{\mathrm{w}}^{(1 / 2) n}\left(R^{n}\right)$-potentials, J. Math. Phys. 22 (1981), 1033-1044.

15. C. Loewner and L. Nirenberg, Differential equations invariant under projective and conformal transformations, Contributions to Analysis [Bers festschrift] (I. Kra et al., eds.), Academic Press, New York, 1975. 
16. V. Nikolaenko, K. Stanyukovich and G. Shikin, Conformal invariance of Weinberg-Salam theories, Theoret. and Math. Phys. 46 (1981), 394-401.

17. B. Ørsted, Conformally invariant differential equations and projective geometry, J. Funct. Anal. 44 (1981), 1-23.

18. R. Penrose, Conformal treatment of infinity, les Houches Summer School, 1963.

19. E. A. Tagirov and I. T. Todorov, A geometric approach to conformally invariant field equations, Lecture Notes in Physics, vol. 80, Springer, Berlin and New York (Rome 1977).

20. N. Trudinger, Some remarks on conformal deformations of Riemannian metrics, Ann. Scuola Norm. Sup. Pisa 22 (1968), 265-274.

21. A. Zee, Broken symmetric theory of gravity, Phys. Rev. Letters 44 (1980), 703-705.

Department of Mathematics, The Johns Hopkins University, Baltimore, Maryland 21218 